\title{
Biomimetic Corrugated Silicon Nanocone Arrays for Self-Cleaning Antireflection Coatings
}

\author{
Yandong Wang ${ }^{1}$, Nan $\mathrm{Lu}^{1}(\bowtie)$, Hongbo Xu ${ }^{1}$, Gang Shi ${ }^{1}$, Miaojun $\mathrm{Xu}^{1}$, Xiaowen $\mathrm{Lin}^{2}$, Haibo $\mathrm{Li}^{1}$, Wentao Wang ${ }^{1}$ \\ Dianpeng Qi ${ }^{1}$, Yanqing $\mathrm{Lu}^{2}$, and Lifeng $\mathrm{Chi}^{1,3}(\bowtie)$ \\ ${ }^{1}$ State Key Laboratory of Supramolecular Structure and Materials, Jilin University, Changchun 130012, China \\ ${ }^{2}$ National Laboratory of Solid State Microstructures, Nanjing University, Nanjing 210093, China \\ ${ }^{3}$ Physikalisches Institut and Center for Nanotechnology (CeNTech), Westfälische Wilhelms-Universität, D-48149 Münster, Germany \\ Received: 28 April 2010 / Revised: 25 May 2010 / Accepted: 25 May 2010 \\ C The Author(s) 2010. This article is published with open access at Springerlink.com
}

\begin{abstract}
Corrugated silicon nanocone (SiNC) arrays have been fabricated on a silicon wafer by two polystyrene-spheremonolayer-masked etching steps in order to create high-performance antireflective coatings. The reflectance was reduced from above $35 \%$ to less than $0.7 \%$ in the range $400-1050 \mathrm{~nm}$, and it remained below $0.5 \%$ at incidence angles up to $70^{\circ}$ at $632.8 \mathrm{~nm}$ for both $s$ - and $p$-polarized light. The fluorinated corrugated SiNC array surface exhibits superhydrophobic properties with a water contact angle of $164^{\circ}$.
\end{abstract}

\section{KEYWORDS}

Antireflection, silicon nancone (SiNC) arrays, reactive ion etching, nanosphere lithography, superhydrophobic

\section{Introduction}

Reduction of optical reflection is important for siliconbased optical and optoelectronic devices, such as solar cells, displays, and light sensors [1-4]. Traditionally, single- or multi-layer interference coatings have been widely utilized to suppress reflection. However, these reduce reflection only at a specific wavelength and at specific angles of incidence (AOI) or are limited by the stability problems induced by adhesiveness and thermal mismatch. Inspired by observations of the corneas of nocturnal insects [5], subwavelength structures (SWS) such as nanocone arrays have been developed as a transition layer to suppress reflection $[6,7]$. These SWS are commonly referred to "moth-eye structures" due to the similar arrays of conical protuberances which reduce reflection by effectively providing a graded transition of the refractive index from air to the substrate [8].

Many techniques based on top-down lithography, such as photolithography [9], electron-beam lithography [10], laser interference lithography [11], and nanoimprint lithography [12] have been used to generate antireflective SWS on Si surfaces. However, these techniques are restricted in terms of practical applications because of the expensive equipment required, and their time-consuming and complicated procedures [5]. Bottom-up self-assembly is a simple and cost-effective alternative way to create submicrometerscale periodic arrays [13]. For example, self-assembled masks composed of colloidal nanospheres [14], metal particles [15-17], or an anodic porous alumina membrane [18] combined with a reactive ion etching (RIE) process have been used for the fabrication of

Address correspondence to Nan Lu, luenan@jlu.edu.cn; Lifeng Chi, chi@uni-muenster.de 
SWS surfaces. In principle, upright tapered structures with high aspect ratio that suppress reflections over a wide spectral bandwidth and AOI can be easily fabricated [18]. It is well known that silicon cones with high aspect ratio result in a gradual increase of the effective refractive index ( $n_{\text {eff }}$ ) from air to the substrate, rather than the large refractive index discontinuity associated with flatter surfaces. However, the fabrication of silicon cones with high aspect ratio requires special etching technology, such as chlorinebased RIE [5] or electron cyclotron resonance plasma etching $[8,19]$. Therefore, a simpler method for producing high-performance antireflection coatings is desirable. Herein, we describe the fabrication of corrugated silicon nanocone (SiNC) arrays on a Si substrate based on the combination of nanosphere lithography and RIE. Light reflection is reduced from above $35 \%$ for the flat substrate to below $0.7 \%$ for the corrugated arrays for wavelengths from 400 to $1050 \mathrm{~nm}$, which covers the solar spectrum that is useful for a silicon-based solar cell $[6,13]$. The reflectance is also suppressed over a wide range of AOI for both s- and p-polarized light.

\section{Experimental}

\subsection{Materials}

All solvents and reagents were of reagent quality and were used without further purification. Ethanol, acetone, chloroform, and toluene were purchased from Guangfu Fine Chemical Research Institute (Tianjin, China) and had the highest available purity. Ultrapure water $(18.2 \mathrm{M} \Omega \cdot \mathrm{cm})$ was used directly from a Millipore System (Marlborough, France). The monodisperse polystyrene (PS) spheres with less than $5 \%$ diameter variation and (heptadecafluoro-1,1,2,2tetrahydrodecyl)triethoxysilane were obtained from Sigma-Aldrich Corporation. The Si wafers [n type (100)] were obtained from Youyan Guigu (Beijing, China).

\subsection{Assembly of polystyrene monolayers}

The monolayers of PS nanospheres were prepared according to Ref. [20]. In brief, $0.2 \mathrm{~mL}$ of a PS nanosphere dispersion in absolute ethanol was dropped on the surface of water in an $8-\mathrm{cm}$ diameter glass tank, and the monolayer was then lifted onto the silicon wafer. PS nanosphere monolayers were obtained by allowing the slides to become dry.

\subsection{Fabrication of SiNC arrays}

The fabrication of SiNC arrays was carried out using a Plasmalab Oxford 80 Plus (ICP65) system (Oxford Instruments $\mathrm{Co} ., \mathrm{UK}$ ) with a gaseous mixture of $\mathrm{SF}_{6}$, $\mathrm{CHF}_{3}$, and $\mathrm{O}_{2}$.

\subsection{Characterization}

Scanning electron microscope (SEM) images were obtained using an environmental scanning electron microscope (ESEM, Model XL 30 ESEM FEG from Micro FEI Philips). The samples were sputtered with a thin layer of $\mathrm{Pt}$ ( $2 \mathrm{~nm}$ in thickness) prior to imaging. UV-visible spectra were recorded on a UV-visible spectrometer (Shimadzu UV3600, Shimadzu, Japan).

\section{Results and discussion}

The fabrication of corrugated SiNC arrays involves the following steps, which are summarized in the left column of Fig. 1: (a) A monolayer of colloidal PS spheres with a diameter of $1.1 \mu \mathrm{m}$ was assembled on a Si surface [20], and was utilized as a shadow mask during the subsequent RIE process; (b) Arrays of blunt cones were created by a RIE process with a gaseous mixture of $\mathrm{SF}_{6}, \mathrm{CHF}_{3}$, and $\mathrm{O}_{2}$, and the residual PS spheres were removed by ultrasonication in toluene, giving cones with a height of about $800 \mathrm{~nm}$ (as shown in Fig. S-1 in the Electronic Supplementary Material (ESM)); (c) Smaller PS spheres with a diameter of $580 \mathrm{~nm}$ or $220 \mathrm{~nm}$ were assembled on the surface of the resulting array of blunt cones; (d) The corrugated SiNC arrays were fabricated after an RIE process with the same gases as mentioned above. The corresponding SEM images in the right column of Fig. 1 show the monolayer of colloidal PS spheres with a diameter of $1.1 \mu \mathrm{m}$ (a), the array of blunt cones (b), the monolayer of $580 \mathrm{~nm}$ PS spheres on the array of blunt cones (c), and the final fabricated corrugated SiNC arrays (d).

In our previous work [21], flat tops and grooves were found on all of the planar SiNC arrays which 


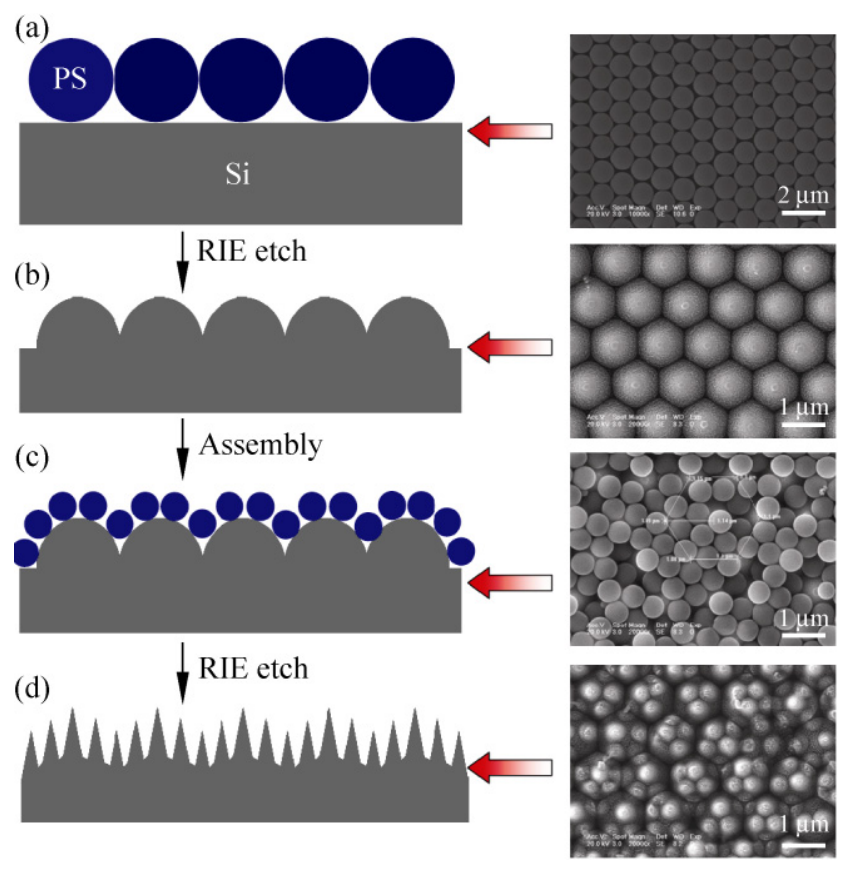

Figure 1 Schematic illustration of the procedure for creating the corrugated SiNC arrays on a Si substrate using PS spheres with a diameter of $1.1 \mu \mathrm{m}$ in (a) and $580 \mathrm{~nm}$ in (c)

were created using a monolayer of PS spheres as a RIE mask on a flat Si wafer. However, the area percentage of flat tops and grooves can be minimized by optimizing the etching conditions, as shown in Figs. 2(a) and 2(b). For PS spheres with a diameter of $580 \mathrm{~nm}$, the height of the silicon cones is about $590 \mathrm{~nm}$, and the diameters of the tip and bottom of the Si cones are about $80 \mathrm{~nm}$ and $560 \mathrm{~nm}$, respectively, so the aspect ratio is around 1.0. In this work, corrugated arrays of cones were created on the Si wafer by progressively reducing the size of the PS spheres in two successive mask-etch processes. The etching conditions were same for fabricating planar SiNC arrays and corrugated SiNC arrays. Figures 2(c) and 2(d) reveal that the corrugated SiNC arrays formed using PS spheres with diameters of $1.1 \mu \mathrm{m}$ and $580 \mathrm{~nm}$ in the first and second steps, respectively, adopt a periodic arrangement. Each silicon pillar has tapered sidewalls just like the protuberances on the cornea of moths, although the heights are different. The height of the silicon cones on the top of the protrusions is in the range $700-800 \mathrm{~nm}$, higher than that in the planar SiNC array. This is because the silicon cones are fabricated on the top of the cones previously fabricated using larger PS spheres. However, the height of the lower silicon cones is lower than those on the top for two reasons: first, the density of reactive species decreases from the top to the bottom of the protrusions during the etching process, resulting in a lower silicon etching rate at the bottom [15]; second, the silicon at the bottom is protected by the overlapping PS spheres. It should be noted that the grooves which existed in the planar SiNC array disappeared after etching, and the center-to-center distance between the neighbouring nanocones is only about $380 \mathrm{~nm}$, which is much shorter than the corresponding distance of $580 \mathrm{~nm}$ in the planar SiNC array (as shown in Fig. S-2 in the ESM). The reason is that the horizontal distance between neighboring $580 \mathrm{~nm}$ PS spheres assembled on the cone arrays is shorter than that on the flat surface. Additionally, the effective thickness of the transition layer is about $1.4 \mu \mathrm{m}$, which is 2.4 times that for the planar SiNC array.

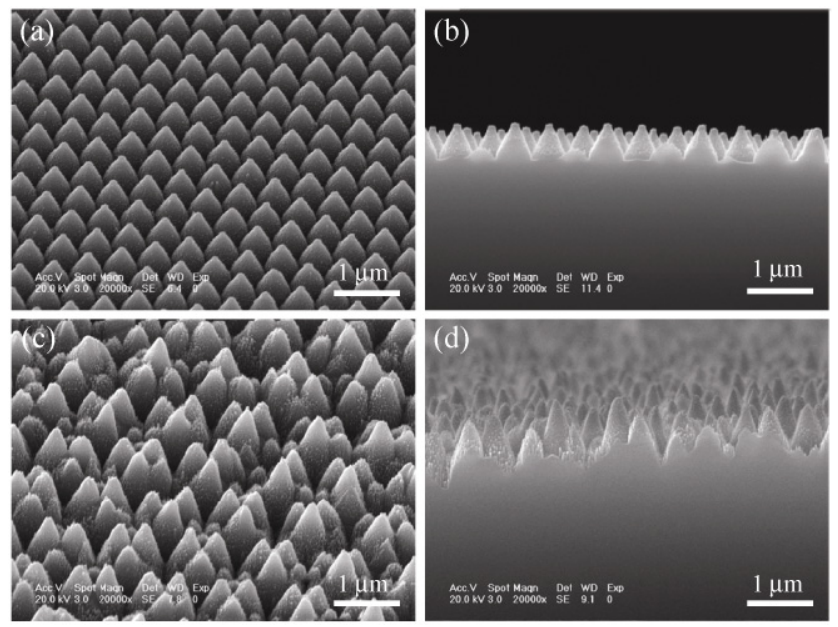

Figure 2 Tilt-view and cross-sectional SEM images of the planar SiNC array formed by using a monolayer of $580 \mathrm{~nm}$ diameter PS spheres on a flat Si surface $(a, b)$ and the corrugated SiNC arrays formed as in Figs. 1 (c) and 1(d)

The specular reflectance was evaluated on a spectrometer attached to standard mirror reflection optics at an incidence angle of $5^{\circ}$. The flat silicon wafer exhibited more than $35 \%$ reflectance in the range 400-1050 nm (the black solid line in Fig. 3(a)) [22]. The reflectance of the planar SiNC array surface was less than $2 \%$ in the same wavelength range (the dashed line in Fig. 3(a)). However, the corrugated SiNC arrays demonstrated even lower reflectance than 
the planar SiNC array over the whole wavelength range. The highest reflectance of the corrugated SiNC arrays was only about $0.7 \%$ (at around $810 \mathrm{~nm}$ ). Furthermore, the reflectance was less than $0.15 \%$ at wavelengths from 400 to $700 \mathrm{~nm}$ (the dotted line in Fig. 3(a)). The specular reflectance increased slightly around $1000 \mathrm{~nm}$ because the indirect band gap of the silicon is around $1100 \mathrm{~nm}$ [8]. A jump in the specular reflectance at around $832 \mathrm{~nm}$ results from the change of grating which occurs at that wavelength. Diffuse reflectance is caused by light scattering by the SWS arrays when their feature size is comparable to or greater than the wavelength of light [8]. Figure 3(b) shows that the hemispherical reflectance of the flat silicon surface in the range 580-900 $\mathrm{nm}$ was reduced from $40 \%$ to $3.3 \%$ or less by forming either planar or corrugated SiNC arrays. The small amount of diffuse reflectance observed might be introduced by the defects in the monolayer of PS spheres. For wavelengths lower than the period of the planar SiNC array $(580 \mathrm{~nm})$, hemispherical reflectance increased with decreasing wavelength due to diffuse reflectance, reaching $10.6 \%$ at $400 \mathrm{~nm}$ (the dashed line in Fig. 3(b)). However, the hemispherical reflectance of corrugated SiNC arrays remained as low as $3.4 \%$ at $500 \mathrm{~nm}$ and only increased slightly to $4.9 \%$ at $400 \mathrm{~nm}$ (the dotted line in Fig. 3(b)). Additionally, the reflectance of the corrugated SiNC arrays at wavelengths above $900 \mathrm{~nm}$ was lower than that of planar SiNC array.

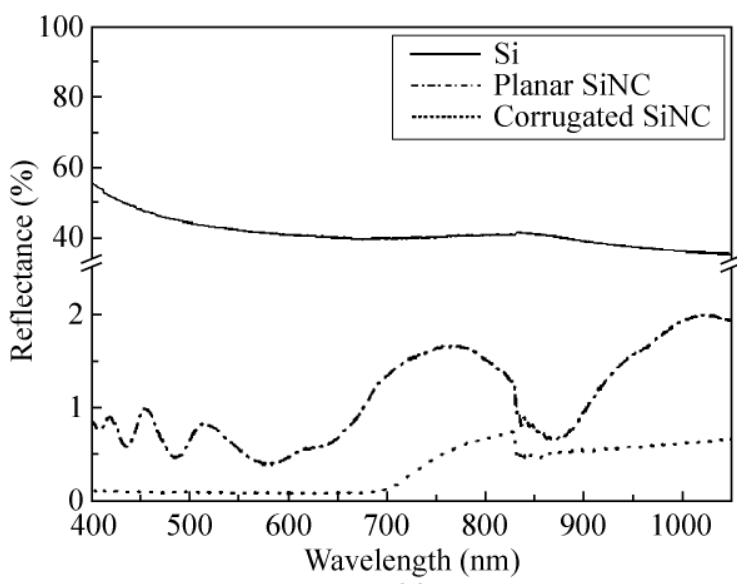

(a)
A good antireflective coating should show low reflectance over a wide AOI for both forms of light polarization $[8,23]$, which is important for applications in sunrise-to-sunset solar cells [24]. The angledependent reflectivities for a polished $\mathrm{Si}$ wafer, planar SiNC array and corrugated SiNC arrays are shown in Fig. 4. These were measured with a He-Ne laser $(632.8 \mathrm{~nm})$ at incident angles from $25^{\circ}$ to $87^{\circ}$ for $p$ - and $s$-polarization, where $p$ and $s$ denote planes of incidence parallel and perpendicular to the electrical field of the light waves, respectively. Figures 4(a) and 4(b) show that the measured specular reflectance of the polished flat $\mathrm{Si}$ wafer varies strongly with AOI for both sand $p$-polarized light. In contrast, both planar and corrugated SiNC arrays are not highly sensitive to polarization. Furthermore, the performance of the corrugated SiNC array is better than that of the planar SiNC array. For both $s$ - and $p$-polarization, the reflectance of the planar SiNC array (the dashed line) reaches a value of $5 \%$ when the $\mathrm{AOI}$ is $70^{\circ}$, while that of the corrugated SiNC arrays remains below $0.5 \%$ at values of AOI ranging from $25^{\circ}$ to $70^{\circ}$ (the dotted line).

The reflectance decreased even more markedly when the corrugated silicon nanowire (SiNW) arrays were formed using $220 \mathrm{~nm}$ PS spheres as the mask in the second step. It is well known that the incident light hardly experiences any change in the dielectric environment if the increment in the refractive indices is sufficiently small. Hence, it is desirable that the

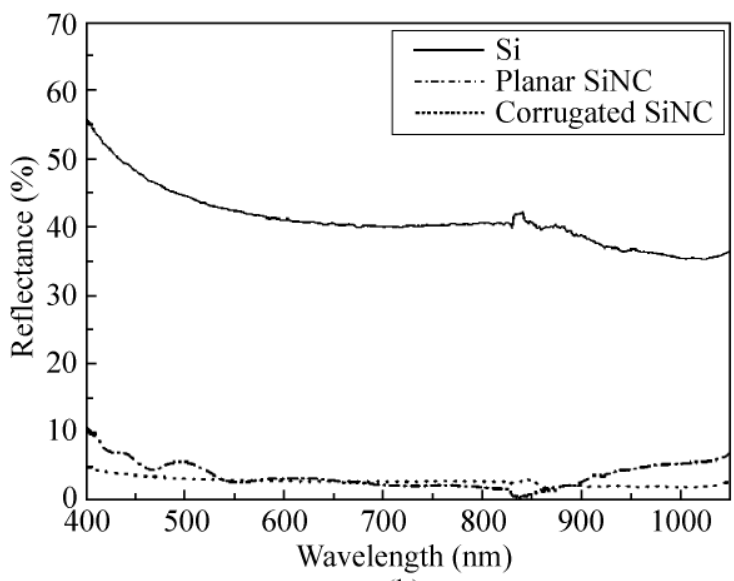

(b)

Figure 3 The (a) specular and (b) hemispherical reflectance of the polished silicon substrate, the planar SiNC array, and the corrugated SiNC arrays fabricated with $580 \mathrm{~nm}$ PS spheres 


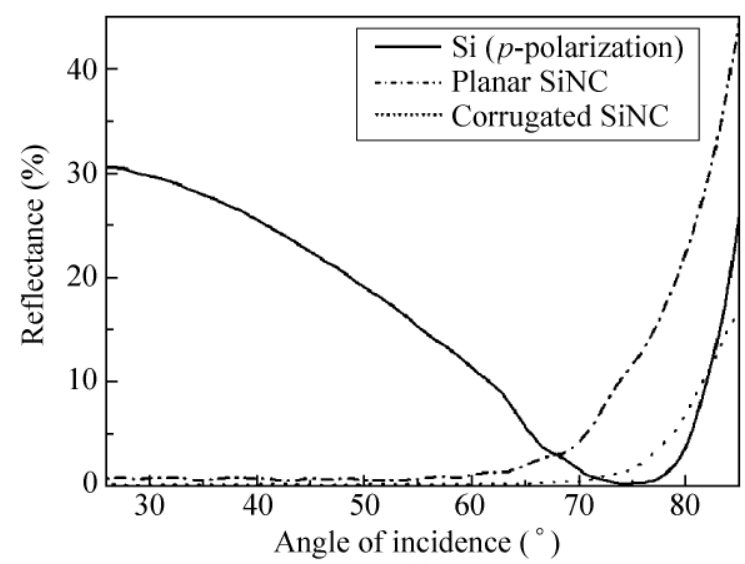

(a)

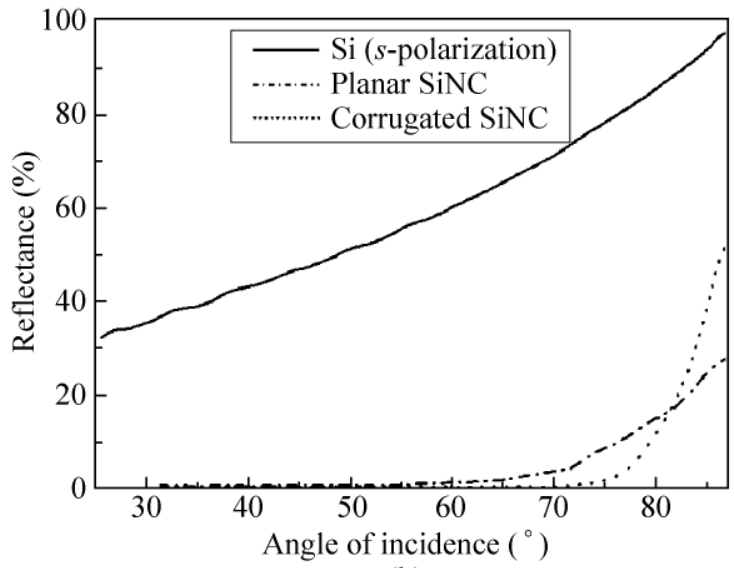

(b)

Figure 4 The dependence of the reflectance on the AOI when the incident light is (a) $p$-polarized, and (b) $s$-polarized

nanopillars are cone-like or spearhead-like in shape of structures, since this gives the required refractive index gradient [25]. In contrast, silicon nanowires with a column-like structure show poor antireflection properties because of the large refractive index discontinuity at the interface with air. As shown in Fig. 5(a), the reflectance of the planar SiNW array formed using $220 \mathrm{~nm}$ diameter PS spheres is above $6 \%$ at most wavelengths and the maximum value is above $10 \%$ at around $500 \mathrm{~nm}$ (the dashed line). The high reflectance can be attributed to the columnar shape and limited aspect ratio. However, the reflectance is reduced to below $0.8 \%$ over the whole measured wavelength range for the corrugated SiNW arrays (the dotted line), and values lower than $0.17 \%$ are even achieved from 400 to $700 \mathrm{~nm}$. The tilt-view SEM images of planar and corrugated SiNW arrays are shown in Figs. 5(b) and 5(c), respectively. When forming the corrugated surface, the assembled $220 \mathrm{~nm}$ PS spheres were not close-packed due to the large size difference
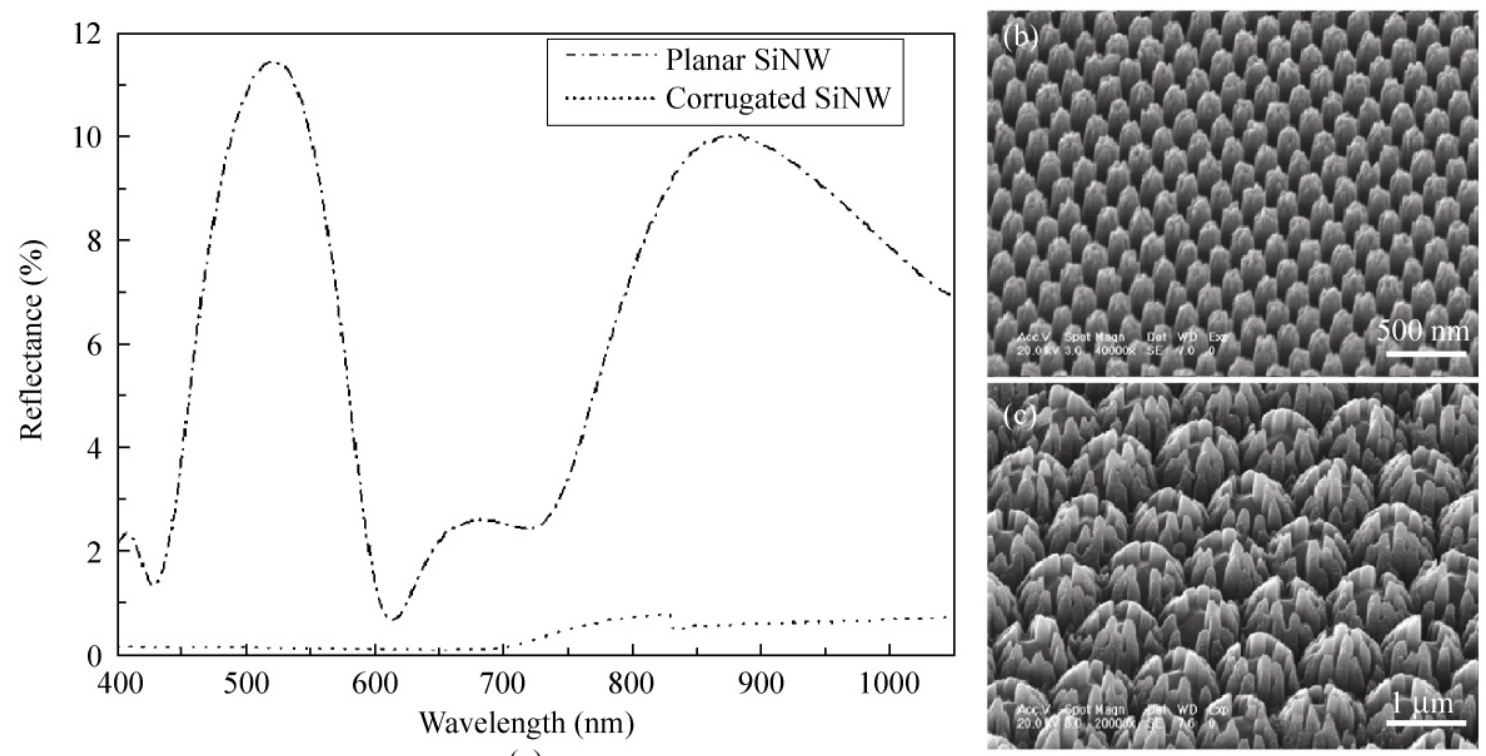

(a)

Figure 5 Comparison of specular reflectance for planar and corrugated SiNW arrays fabricated with 220 nm diameter PS spheres (a), tilt-view SEM images for the planar SiNW array (b), and the corrugated SiNW arrays (c) 
between the PS spheres and the $800 \mathrm{~nm}$ silicon cones on the pre-structured surface; this aspect could be improved by decreasing the height of the blunt cone arrays on the pre-structured surface.

From the viewpoint of effective medium theory [26], the SWS can be considered as an inserted layer with gradient $n_{\text {eff }}$ which changes from the boundary of air/SWS to the boundary of SWS/Si. Reflections occur at each depth of SWS because of the gradient change, and the reflected beams that are out of phase can interfere destructively, thereby reducing the overall reflectance [27]. The $n_{\text {eff }}$ of the corrugated SiNC arrays changes more gradually than that of the planar SiNC array because its effective layer $(1.4 \mu \mathrm{m})$ is thicker than that of the planar SiNC array $(590 \mathrm{~nm})$. For an ideal SWS with near zero reflectance, the $n_{\text {eff }}$ value should gradually increase from 1 to $n_{\mathrm{Si}}$ [18]. This requires SWS with a perfectly tapered shape and "V" shape profiles of the grooves [18, 21]. As revealed by SEM images, some flat tops and grooves are found on the planar SiNC arrays, which induce abrupt increases of $n_{\text {eff }}$ at the air/top and air/groove interfaces. However, when the silicon nanocones are in a corrugated arrangement, the area percentage of the flat tops is dramatically decreased and the grooves disappear. This is why the corrugated SiNC arrays exhibit better antireflection performance than the planar SiNC arrays. Additionally, to avoid scattering from the optical interface, the structural dimension at an optical interface has to be smaller than the wavelength of the incident light [28]. Unfortunately, the fabrication difficulty increases as the feature size becomes smaller. In this work, the fabrication of small features is simply realized by two successive PS monolayer mask-etch processes. Theoretical calculations were carried out by using the rigorous coupled-wave analysis (RCWA) model proposed by Moharam [29], where the reflectance is obtained as a sum of the reflected diffraction efficiencies of different diffraction orders. The calculation model was constructed based on the observation of the fabricated SWS surface by SEM. Figure S-3 in the ESM shows that the calculated trends agree well with the measured results shown in Fig. 3(b).
Additionally, modification of the surface of the corrugated SiNC arrays with the fluoroalkylsilane (heptadecafluoro-1,1,2,2-tetrahydrodecyl)triethoxysil ane produces materials which exhibit excellent superhydrophobic properties [30] with a water contact angle about $164^{\circ}$ (Fig. S-4 in the ESM), which show promise for the development of self-cleaning antireflection coatings for crystalline silicon solar cells [31].

\section{Conclusions}

We have developed a technique for fabricating corrugated SiNC arrays which exhibit good antireflection and superhydrophobic properties. The effective thickness of the transition layer is enlarged by the compound silicon pillars, which can avoid strict requirements such as sharp conical shape and high aspect ratio. Small-sized features can also be simply created using this technique.

\section{Acknowledgements}

This work was supported by the National Natural Science Foundation of China (No. 20373019), the Program for New Century Excellent Talents in University, and the National Basic Research Program (Nos. 2007CB808003 and 2009CB939701).

Electronic Supplementary Material: Figures S-1-S-4 showing cross-sections and measured reflectance of blunt cone arrays with $1.1 \mu \mathrm{m}$ diameter PS spheres as an etching mask, the calculation of reduced feature size, reflectance of corrugated SiNC arrays simulated with the RCWA model, water contact angles of the polished $\mathrm{Si}$, planar and corrugated SiNC arrays surfaces, are available in the online version of this article at http://dx.doi.org/10.1007/s12274-010-0012-x and are accessible free of charge.

Open Access: This article is distributed under the terms of the Creative Commons Attribution Noncommercial License which permits any noncommercial use, distribution, and reproduction in any medium, provided the original author(s) and source are credited. 


\section{References}

[1] Striemer, C. C.; Fauchet, P. M. Dynamic etching of silicon for broadband antireflection applications. Appl. Phys. Lett. 2002, 81, 2980-2982.

[2] Lee, Y. J.; Ruby, D. S.; Peters, D. W.; McKenzie, B. B.; Hsu, J. W. P. ZnO nanostructures as efficient antireflection layers in solar cells. Nano Lett. 2008, 8, 1501-1505.

[3] Gombert, A.; Glaubitt, W.; Rose, K.; Dreibholz, J.; Blasi, B.; Heinzel, A.; Sporn, D.; Doll, W.; Wittwer, V. Antireflective transparent covers for solar devices. Sol. Energy 2000, 68, 357-360.

[4] Lee, C.; Bae, S. Y.; Mobasser, S.; Manohara, H. A novel silicon nanotips antireflection surface for the micro sun sensor. Nano Lett. 2005, 5, 2438-2442.

[5] Min, W. L.; Jiang, B.; Jiang, P. Bioinspired self-cleaning antireflection coatings. Adv. Mater. 2008, 20, 3914-3918.

[6] Zhu, J.; Yu, Z. F.; Burkhard, G. F.; Hsu, C. M.; Connor, S. T.; Xu, Y. Q.; Wang, Q.; McGehee, M.; Fan, S. H.; Cui, Y. Optical absorption enhancement in amorphous silicon nanowire and nanocone arrays. Nano Lett. 2009, 9, 279-282.

[7] Boden, S. A.; Bagnall, D. M. Tunable reflection minima of nanostructured antireflective surfaces. Appl. Phys. Lett. 2008, 93, 133108 .

[8] Huang, Y. F.; Chattopadhyay, S.; Jen, Y. J.; Peng, C. Y.; Liu, T. A.; Hsu, Y. K.; Pan, C. L.; Lo, H. C.; Hsu, C. H.; Chang, Y. H.; Lee, C. S.; Chen, K. H.; Chen, L. C. Improved broadband and quasi-omnidirectional anti-reflection properties with biomimetic silicon nanostructures. Nat. Nanotechnol. 2007, 2, 770-774.

[9] Heine, C.; Morf, R. H. Submicrometer gratings for solar energy applications. Appl. Opt. 1995, 34, 2476-2482.

[10] Kanamori, Y.; Roy, E.; Chen, Y. Antireflection subwavelength gratings fabricated by spin-coating replication. Microelectron. Eng. 2005, 78-79, 287-293.

[11] Aydin, C.; Zaslavsky, A.; Sonek, G. J.; Goldstein, J. Reduction of reflection losses in $\mathrm{ZnGeP}_{2}$ using motheye antireflection surface relief structures. Appl. Phys. Lett. 2002, 80, 2242-2244.

[12] Zhang, G. M.; Zhang, J.; Xie, G. Y.; Liu, Z. F.; Shao, H. B. Cicada wings: A stamp from nature for nanoimprint lithography. Small 2006, 2, 1440-1443.

[13] Sun, C. H.; Jiang, P.; Jiang, B. Broadband moth-eye antireflection coatings on silicon. Appl. Phys. Lett. 2008, 92, 061112.
[14] Chen, H. L.; Chuang, S. Y.; Lin, C. H.; Lin, Y. H. Using colloidal lithography to fabricate and optimize subwavelength pyramidal and honeycomb structures in solar cells. Opt. Express 2007, 15, 14793-14803.

[15] Lohmuller, T.; Helgert, M.; Sundermann, M.; Brunner, R.; Spatz, J. P. Biomimetic interfaces for high-performance optics in the deep-UV light range. Nano Lett. 2008, 8, 1429-1443.

[16] Wang, S.; Yu, X. Z.; Fan, H. T. Simple lithographic approach for subwavelength structure antireflection. Appl. Phys. Lett. 2007, 91, 061105.

[17] Lee, Y.; Koh, K.; Na, H.; Kim, K.; Kang, J. J.; Kim, J. Lithography-free fabrication of large area subwavelength antireflecion structure using thermally dewetted $\mathrm{Pt} / \mathrm{Pd}$ alloy etch mask. Nanoscale Res. Lett. 2009, 4, 364-370.

[18] Kanamori, Y.; Hane, K.; Sai, H.; Yugami, H. 100 nm period silicon antireflection structures fabricated using a porous alumina membrane mask. Appl. Phys. Lett. 2001, 78, 142.

[19] Ting, C. J.; Huang, M. C.; Tsai, H. Y.; Chou, C. P.; Fu, C. C. Low cost fabrication of the large-area anti-reflection films from polymer by nanoimprint/hot-embossing technology. Nanotechnology 2008, 19, 205301-205305.

[20] Rybczynski, J.; Hilgendorff, M.; Giersig, M. Nanosphere lithography-Fabrication of various periodic magnetic particle arrays using versatile nanosphere masks. In Low-Dimensional Systems: Theory, Reparation, and Some Applications (NATO Science Series II: Mathematics, Physics and Chemistry, Vol. 91). Liz-Marzán, L. M.; Giersig, M., Eds.; Springer: Berlin, 2003; pp 163-172.

[21] Xu, H. B.; Lu, N.; Qi, D. P. ; Hao, J. Y.; Gao, L. G.; Zhang, B.; Chi, L. F. Biomimetic antireflective Si nanopillar arrays. Small 2008, 4, 1972-1975.

[22] Lalanne, P.; Morris, G. M. Antireflection behavior of silicon subwavelength periodic structures for visible light. Nanotechnology 1997, 8, 53-56.

[23] Kanamori, Y.; Sasaki, M.; Hane, K. Broadband antireflection gratings fabricated upon silicon substrates. Opt. Lett. 1999, 24, 1422-1424.

[24] Chen, Q.; Hubbard, G.; Shields, P. A.; Liu, C.; Allsopp, D. W. E.; Wang, W. N.; Abott, S. Broadband moth-eye antireflection coatings fabricated by low-cost nanoimprinting. Appl. Phys. Lett. 2009, 94, 263118.

[25] Chiu, C. H.; Yu, P. C.; Kuo, H. C.; Chen, C. C.; Lu, T. C.; Wang, S. C.; Hsu, S. H.; Cheng, Y. J.; Chang, Y. C. Broadband and omnidirectional antireflection employing 
disordered GaN nanopillars. Opt. Express 2008, 16, 8748-8754.

[26] Yu, Z. N.; Gao, H.; Wu, W.; Ge, H. X.; Chou, S. Y. Fabrication of large area subwavelength antireflection structures on Si using trilayer resist nanoimprint lithography and liftoff. J. Vac. Sci. Technol. B 2003, 21, 2874-2877.

[27] Hadobas, K.; Kirsch, S.; Carl, A.; Acet, M.; Wassermann, E. F. Reflection properties of nanostructure-arrayed silicon surfaces. Nanotechnology 2000, 11, 161-164.

[28] Li, X.; Gao, J. P.; Xue, L. J.; Han, Y. C. Porous polymer films with gradient-refractive-index structure for broadband and omnidirectional antireflection coatings. Adv. Funct. Mater. 2010, 20, 259-265.
[29] Moharam, M. G.; Pommet, D. A.; Gram, E. B.; Gaylord, T. $\mathrm{K}$. Formulation for stable and efficient implementation of the rigorous coupled-wave analysis of binary gratings. $J$. Opt. Soc. Am. A 1995, 12, 1068-1076.

[30] Cao, M. W.; Song, X. Y.; Zhai, J.; Wang, J. B.; Wang, Y. L. Fabrication of highly antireflective silicon surfaces with superhydrophobicity. J. Phys. Chem. B 2006, 110, 13072-13075.

[31] Li, Y. F.; Zhang, J. H.; Zhu, S. J.; Dong, H. P.; Wang, Z. H.; Sun, Z. Q.; Guo, J. R.; Yang, B. Bioinspired silicon hollowtip arrays for high performance broadband. J. Mater. Chem. 2009, 19, 1806-1810. 